\title{
UNA ESPECIE NUEVA DE HYMENOPHYLLUM Y UNA VARIEDAD NUEVA DE TRICHOMANES COLLARIATUM BOSCH (FILICALES: HYMENOPHYLLACEAE) EN COSTA RICA
}

\author{
Alexander Fco. Rojas Alvarado \\ Universidad de Costa Rica, Sede de Occidente. Apdo. 111-4250, San Ramón, Alajuela. \\ Dirección actual: Museo Nacional de Costa Rica, Depto. de Historia Natural \\ Apdo. 749-1000, San José, Costa Rica. afrojasa@hotmail.com
}

\begin{abstract}
A new species and a new variety of Hymenophyllaceae are described for Costa Rica. Hymenophyllum talamancanum, somewhat similar to H. horizontale C. V. Morton, and Trichomanes collariatum Bosch var. alvaradoi, with some differences compared to the typical variety.
\end{abstract}

RESUMEN. Se describen una nueva especie y una nueva variedad de Hymenophyllaceae de Costa Rica: Hymenophyllum talamancanum, parecido a H. horizontale C.V. Morton, y Trichomanes collariatum Bosch var. alvaradoi, con algunas diferencias respecto a la variedad típica.

Key words / Palabras Clave: Pteridophyta, Hymenophyllaceae, Hymenophyllum, Trichomanes, Costa Rica

Según Lellinger (1989), en Costa Rica habitan 29 especies de Hymenophyllum y 38 especies y una subespecie de Trichomanes. Posteriormente, el mismo autor (1991) agrega H. trichomanoides Bosch y H. decurrens (Jacq.) Sw. Pacheco (1995) incluye 28 especies de Hymenophyllum y 40 especies, una variedad y una subespecie de Trichomanes en Costa Rica; en este trabajo tres nombres de Hymenophyllum utilizados por Lellinger (1991) fueron reducidos a sinónimos. Para dos especies más, Pacheco utiliza los nombres más antiguos y registra, además, $H$. lanatum Fée y $H$. trapezoidale Liebm. en la pteridoflora de Costa Rica. Adicionalmente, él registra Trichomanes botryoides Kaulf. y T. rhipidophyllum Sloss. y trata a T. cocos H. Christ como una variedad de T. capillaceum L. Rojas (1996a) informa la presencia de Hymenophyllum hemidimorphum R.C. Moran \& B. Øllg., Trichomanes delicatum Bosch y T. plumosum Kunze; también describe Hymenophyllum cocosense (Rojas 1996b), una especie endémica en la Isla del Coco, Costa Rica. Rojas (2001) corrige el informe de Trichomanes plumosum, que es T. micayense Hieron., y Pacheco (2002) describe T. ribae de Costa Rica y Panamá. En el presente trabajo se describen una nueva especie y una nueva variedad de Costa Rica.
Estos nuevos táxones han sido así considerados después de comparar con especies afines en distintos tratamientos taxonómicos de Mesoamérica, el Caribe y Sudamérica (Proctor 1985, Mickel \& Beitel 1988, Lellinger 1989, Proctor 1989, Tryon \& Stolze 1989, Moran \& Riba 1995, Smith 1995), en publicaciones recientes que incluyen a la familia Hymenophyllaceae (Lellinger 1985, 1991, 1994, Moran \& Øllgaard 1995, Rojas 1996a, 1996b, Moran \& Øllgaard 1998, Moran 2000, Pacheco 2002) y después de revisar las colecciones del Herbario Nacional de Costa Rica (CR) y del Instituto Nacional de Biodiversidad (INB).

Hymenophyllum talamancanum A. Rojas, sp. nova TIPO: Costa Rica. Limón: Talamanca, Bratsi, Amubri, Alto Lari, siguiendo la fila entre Río Depari y Río Lari, 9²5’30”N, 8303’35”'W, 450 m, 3 mar 1992, G. Herrera 5145 (Holotipo: INB, isotipos: CR, MO, UC). Los isotipos son mixtos con $H$. sieberi.

FIG. 1.

A H. horizontalis C.V. Morton rhachi absque alata, pinnis magis divisis, pilis rhacheos, costae venaesque sessilis densis, necnon segmentis angustioris longioribus differt.

Epífita; rizoma de 0.3-0.4 mm de diámetro, rep- 


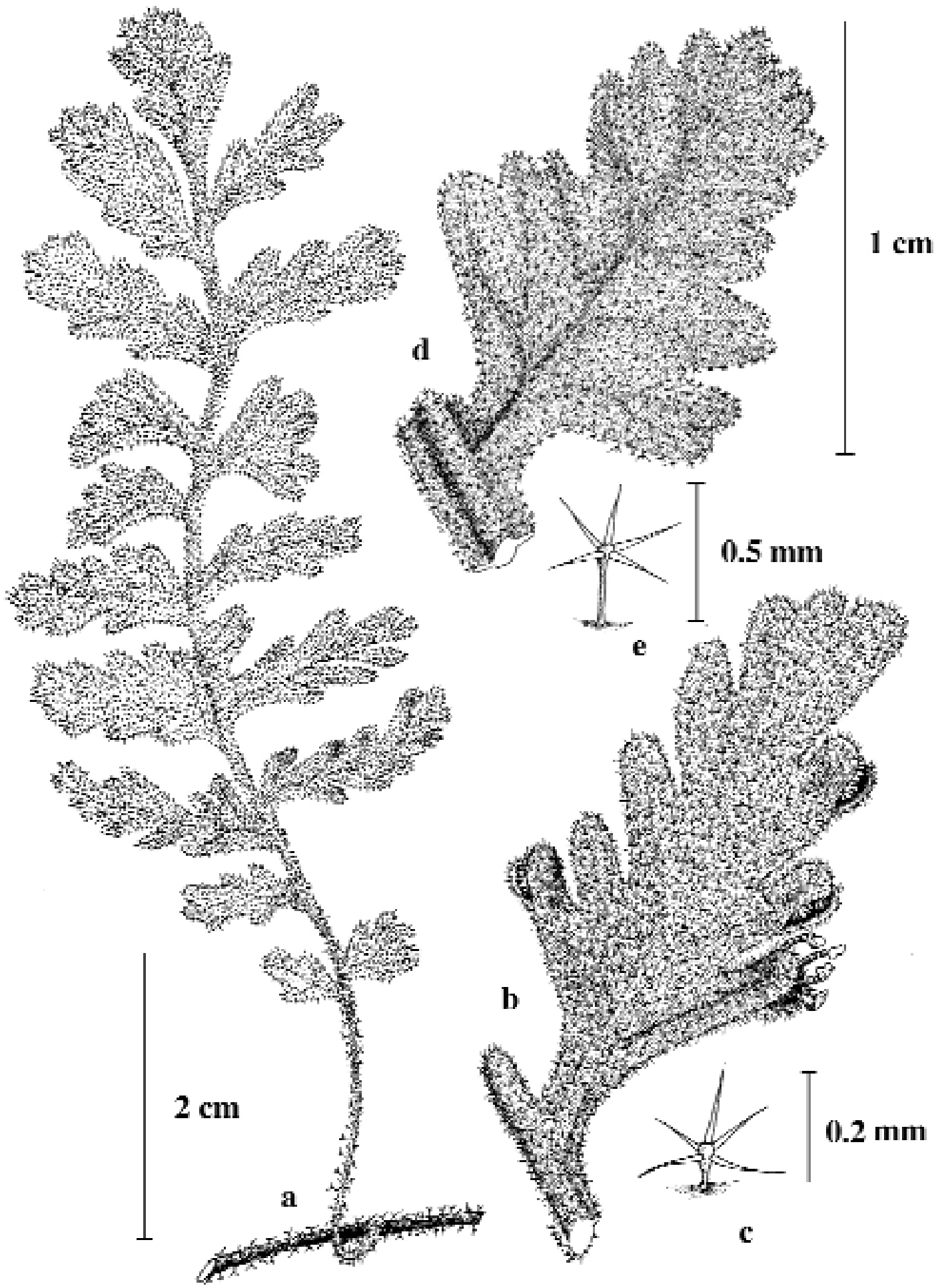

Fig. 1. Hymenophyllum talamancanum A. Rojas (G. Herrera 5145, herbario): a) Fronda, b) Pinna, c) Pelo de la lámina. H. sieberi (C. Presl) Bosch: d) Pinna, e) Pelo de la lámina. 
tante; pelos del rizoma (0.5-) 1-2 mm de largo; frondas 6-22 cm de largo, de crecimiento indeterminado, pendulosas, comúnmente separadas por $1.5-2 \mathrm{~cm}$; pecíolo 0.6-4.3 cm de largo, 0.3-0.4 $\mathrm{mm}$ de diámetro, no alado; raquis lineal a flexuoso, levemente alado a raramente exalado, reduciéndose hacia la base entre las pinnas (como base decurrente de las pinnas), el ala 0.1-0.3 (-0.5) $\mathrm{mm}$ de ancho, con pelos estrellados de 3-6 rayos, sésiles a más comúnmente cortamente pediculados; lámina 5-20 x 1.5-3 cm, pinnado-pinnatífida a bipinnatisecta, elíptica a linear-elíptica, reduciéndose si ensanchándose por regiones, con las pinnas basales reducidas y un ápice obtuso e indeterminado; pinnas 7-20 × 3-6 $\mathrm{mm}$, angostamente deltadas, planas, decurrentes en el lado basiscópico, sésiles o incisas en el lado acroscópico, densamente pelosas en el margen, las venas y el tejido laminar, los pelos ca. $0.2 \mathrm{~mm}$ de largo, estrellados con 3-6 rayos, sésiles o escasamente pedicelados; segmentos 0.7-1.5 mm de ancho, simples (muy raramente bifurcados), el segmento basal acroscópico comúnmente doble, (3-) 4-10 por pinna; involucro de 0.6-0.8 (-1) $\mathrm{mm}$ de diámetro, ovado a redondo, inmerso en la lámina, igual de ancho que el segmento, a lo largo de toda la fronda, de (3-) 5-10 (-18) por pinna; receptáculo no exerto.

ETIMOlOGíA. El epíteto talamancanum se refiere a la única localidad donde se ha hallado la especie, el cantón de Talamanca, provincia de Limón.

DistriBUCiÓN: Se conoce sólo el ejemplar tipo de Talamanca, a $450 \mathrm{~m}$.

Hymenophyllum talamancanum es muy similar a $H$. horizontale C.V. Morton, pero se puede distinguir fácilmente de éste por presentar raquis no alado o alado sólo por las pinnas decurrentes ( $v s$. alado), pinnas más pequeñas $(0.7-2 \times 0.3-0.6 \mathrm{~cm} v s .1 .5-4.5$ x 0.6-1 cm) y más incisas, y pelos de la lámina sésiles a escasamente pediculados (vs. pediculados). Se diferencia de H. sieberi (C. Presl) Bosch por la lámina densamente setosa ( $v s$. dispersamente setosa) con pelos sésiles a cortamente pediculados (vs. pediculados), raquis no alado o alado sólo por las pinnas decurrentes (vs. alado) y pinnas más pequeñas $(0.7-2 \times 0.3-0.6 \mathrm{~cm}$ vs. $1.6-5 \times 0.9-1.8 \mathrm{~cm})$. La planta parece intermedia entre $H$. horizontale y
H. plumosum Kaulf., pero las esporas son bien formadas (regulares), por lo que se descarta la idea de un híbrido. Difiere de Hymenophyllum plumosum por las frondas distantes $1.5-2 \mathrm{~cm}(v s .2-5 \mathrm{~cm})$, raquis levemente alado ( $v s$. no alado), pinnas fuertemente pinnatífidas (incisas _o más entre el margen y la costa) a pinnatisectas ( $v s$. levemente pinnatífidas con incisión no más de _ entre el margen y la costa), pinnas basales sésiles (vs. libres), lámina densamente pelosa con los tricomas próximos (vs. medianamente pelosa con los tricomas separados) y los tricomas por lo común cortamente peciolados (vs. siempre sésiles).

Muy a menudo las muestras de herbario de Hymenophyllaceae epífitas son mixtas, con dos o más especies; incluso pueden ser intergenéricas, principalmente cuando el recolector no es especializado en este grupo, lo que refleja un problema parecido al que se presenta en recolectas de Bryophyta. Esto no se debe a la variabilidad de las especies, sino a la capacidad de muchas de ellas de asociarse naturalmente. Los isotipos de la especie nueva son muestras mixtas, pero morfológicamente diferentes y separables entre sí, como se ha anotado en este escrito. Para evitar confusiones, el holotipo ya fue separado y está representado sólo por la especie nueva descrita aquí.

Trichomanes collariatum Bosch var. alvaradoi A. Rojas, var. nova

TIPO: Costa Rica. Puntarenas: Puntarenas, Parque

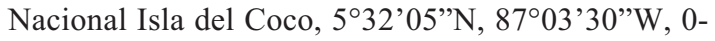
100 m, 22 jun 1997, A. Rojas 3651 (Holotipo: INB, isotipos: AAU, CR, MO, NY, UAMIZ, UC, US).

FIG. 2.

A varieta collariata frondibus brevioribus, stipite longioris, lamina breviori lanceolata differt.

Planta epipétrica, raramente hemiepífita; rizoma 0.5-2 $\mathrm{mm}$ de diámetro, rastrero, verde a pardo, los tricomas abundantes, segmentados, flexuosos, pardos a negruzcos; frondas 7-20 x 4-10 cm, monomorfas, distantes; pecíolo verde, pajizo a pardo, brillante, $4-10 \times 0.05-0.1 \mathrm{~cm}, 1 / 3-1 / 5$ de la longitud de la fronda, subterete, alado, el ala $0.5-1 \mathrm{~mm}$ de ancho, verde, pelosa, los escasos tricomas seme- 


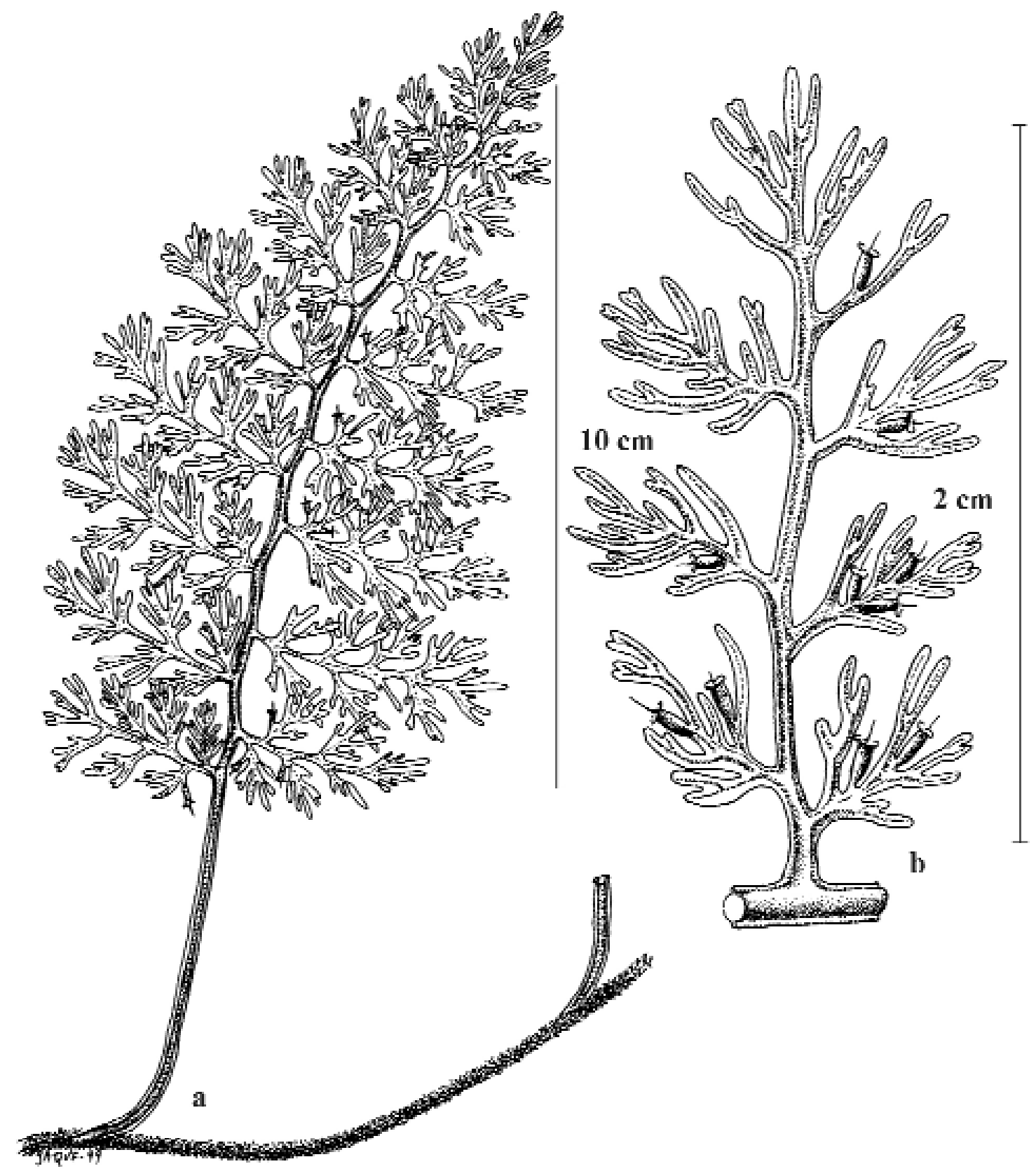

Fig. 2. Trichomanes collariatum Bosch var. alvaradoi A. Rojas (A. Rojas 3651, herbario): a) Hábito, b) Pinna.

jantes a los del rizoma; lámina glabra, verde claro a verde oscuro, $6-15 \times 2.5-7 \mathrm{~cm}$, lanceolada a lanceolado-oblonga, bi- a tripinnatífida, membranácea, la base abruptamente reducida, el ápice obtuso a agudo, pinnatífido; raquis verde, muy dispersamente peloso, los tricomas ca. $0.1 \mathrm{~mm}$, a menudo glandu- lares, alado, las alas $0.2-1 \mathrm{~mm}$ de ancho, verdes, amarillentas a pardas; pinnas 5-15 pares, 1-3.5 x 0.5$2 \mathrm{~cm}$, ovadas a lanceoladas, oblongas, inequiláteras, las pínnulas basales acroscópicas más desarrolladas, en ángulo recto a ascendentes; últimos segmentos 0.4-0.7 mm de ancho, oblongos, glabros, los már- 
genes enteros, el ápice redondeado, simple o bífido; nervadura abierta, anádroma, pinnada; nervaduras falsas ausentes; células pequeñas y no translúcidas; soros 1-12 por pinna, presentes en la axila de los segmentos, 1 por pínnula (o a veces dos en la pínnula basal acroscópica); involucro 1.8-3 x 0.5-1 mm, infundibuliforme, exerto, marginado, el ala ca. 0.1 $\mathrm{mm}$, claramente bilabiado, con los lóbulos 0.2-0.5 x 1-1.3 mm, muy abiertos, el margen sin células oscuras; receptáculo cortamente exerto, filiforme; esporangios 10-18 por soro.

Etimología. Esta variedad es dedicada al finado Joaquín Alvarado, exdirector del Área de Conservación Isla del Coco, en homenaje a su buena labor por la conservación de dicho territorio y por su colaboración en mi expedición a la isla.

DistRIBUCIÓN: Variedad conocida sólo de la Isla del Coco a 460-630 m.

Paratipos: Costa Rica. Puntarenas: Puntarenas, Parque Nacional Isla del Coco, Bahía Iglesias, 0-50 m, mar 1970, L. Gómez 3318 a (CR), L. Gómez $3345 a$ (CR); cuenca superior del Valle Wafer, 200 m, mar 1974, L. Gómez 4540 (CR), L. Gómez 4543 (CR); región inferior del Valle Wafer, 0-100 m, mar 1974, L. Gómez 4547 (CR); Bahía Iglesias, 0-50 m, febr 1976, L. Gómez 6557 (CR); between Chatham and Waffer Bays, 100-232 m, dic 1981, L. Gómez 18073 (CR); Chatham Bay, Quebrada Liebre, 50-75 m, 15 abr 1965, A. Jiménez 3208 (CR); Bahía

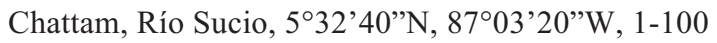
m, 17 oct 1994, F. Quesada 1116 (INB, MO); Río Genio, 20 ene 1980, P. Sánchez 29 (CR); Parque Nacional Isla del Coco, $5^{\circ} 32.5^{\prime} \mathrm{N}, 87^{\circ} 02.4^{\prime} \mathrm{W}$, nov 2002, J. Trusty \& H. Kessler 517 (CR); Isla de Cocos, dic 1939, J. Valerio 1089 (CR).

Trichomanes collariatum Bosch var. alvaradoi difiere de la variedad típica por frondas $7-20 \mathrm{~cm}$ de largo [vs. (15-) 25-60 cm], pecíolo (2/3-) 1/3-1/5 de la longitud de la fronda [vs. 1/6-1/20 (-1/50) de la longitud de la fronda], lámina $6-15 \times 2.5-7 \mathrm{~cm}[v s$. (12-) $20-55 \times(5-) 7-15 \mathrm{~cm}$ ], lanceolada a lanceolado-oblonga ( $v s$. lanceolado-oblonga a linear-elíptica), abruptamente reducida en la base ( $v s$. gradualmente reducida en la base), 5-15 pares de pinnas ( $v s$. 15-35 pares de pinnas), soros 1-12 por pinna (vs. 3-
$28(-50)$ por pinna), soros 1 por pínnula, excepto 2 en la pínnula basal acroscópica (vs. 1-6 por pínnula).

Gómez (1975) indica en su lista que Trichomanes collariatum Bosch tiene frondas hasta de $40 \mathrm{~cm}$ de largo, pero ninguna de las muestras de herbario provenientes de la Isla del Coco tiene tal longitud; además, todas las muestras citadas por Gómez (1975) son estériles. Como se indicó anteriormente, esta variedad de la Isla del Coco no sobrepasa $20 \mathrm{~cm}$ de largo. El material determinado por Gómez (L. D. Gómez 3318a, CR, F, US) como $T$. diaphanum Kunth representa, en realidad, el estado juvenil de $T$. collariatum var. alvaradoi.

Agradecimientos. Al Museo Nacional de Costa Rica por permitirme utilizar mi tiempo laboral en la corrección del artículo, a los herbarios consultados por permitirme utilizar sus colecciones, a los revisores anónimos por sus comentarios, que apoyaron la hipótesis de este estudio, y a Francisco Quesada por sus excelentes ilustraciones.

\section{LiTERATURA CITADA}

Gómez, L. D. 1975. Contribuciones a la Pteridología Costarricense. VII. Pteridofitos de la Isla de Cocos. Brenesia 6: 33-48.

Lellinger, D.B. 1985. Nomenclatural and taxonomic notes on the pteridophytes of Costa Rica, Panama and Colombia, II. Proc. Biol. Soc. Wash. 98(2): 366-390.

Lellinger, D.B. 1989. The ferns and fern-allies of Costa Rica, Panama and the Chocó. Part I. Pteridologia 2: 185-228.

Lellinger, D.B. 1991. Notes on Neotropical Hymenophyllaceae. Amer. Fern J. 81(1): 24-37.

Lellinger, D. B. 1994. Trichomanes polypodioides and its allies. Amer. Fern J. 84(1): 1-4.

Mickel, J. T. \& J.M. Beitel. 1988. Pteridophyte Flora of Oaxaca, México. Mem. New York Bot. Gard. 46: 1568.

Moran, R.C. 2000. Trichomanes resinosum (Hymenophyllaceae), a new species from southern Venezuela and adjacent Guyana. Brittonia 52(3): 238-240.

Moran, R.C. \& B. Øllgaard. 1995. Six new species of ferns (Polypodiopsida) from Ecuador. Nord. J. Bot. 15(2): 177-185.

Moran, R.C. \& B. Øllgaard. 1998. New species of ferns (Polypodiopsida) from Ecuador. Nord. J. Bot. 18(4): 431-439.

Pacheco, L. 2002. Trichomanes ribae (Hymenophyllaceae), a new filmy fern from Costa Rica and Panama. Amer. Fern J. 92(4): 294-295. 
Proctor, G.R. 1985. Ferns of Jamaica, a guide to the Pteridophytes. British Museum. 631 p.

Proctor, G.R. 1989. Ferns of the Puerto Rico and the Virgin Islands. Mem. New York Bot. Gard. 53: 1-389.

Rojas, A.F. 1996a. Aportes a la Flora Pteridophyta Costarricense. I. Informes. Brenesia 45-46: 1-6.

Rojas, A.F. 1996b. Aportes a la Flora Pteridophyta Costarricense. II. Taxones nuevos. Brenesia 45-46: 3350.

Rojas, A. F. 2001. Seis especies nuevas y dos nuevos re- gistros de helechos (Pteridophyta) para Costa Rica. Rev. Biol. Trop. 49: 435-452.

Smith, A.R. 1995. Hymenophyllaceae. In: P.E. Berry, B.K. Holst \& K. Yatskievych (eds.). Flora of the Venezuelan Guayana. Vol. 2. Pteridophytes, Spermatophytes, Acanthaceae-Araceae. Timber Press. Oregon, p. 159-185.

Tryon, R.M. \& R.G. Stolze. 1989. Pteridophyta of Perú. Part I. 1. Ophioglossaceae - 12. Cyatheaceae. Fieldiana, Bot., n.s. 20: 1... 